\title{
Solving the Dynamic Lot-Sizing Problem with Safety Stocks and Limited Inventories based on New Properties
}

\author{
Shaorui $\mathrm{LI}^{*}$ and Mikio KUBO**
}

\begin{abstract}
The dynamic lot-sizing problem with safety stocks and limited inventories is often met in practice. The goal of the problem aims at determining the relative frequency of setups satisfying the demands under restrictions of the safety stock and limited inventory over a planning horizon at minimum cost. In this paper a framework to solve this problem effectively is proposed, which consists of two procedures devised to deal with the safety stock and limited inventory due to new valid inequalities, and new properties to obtain optimal policies. Based on these properties a dynamic programming algorithm is introduced and then a priori is added to the branch-and-bound tree of the reduced shortest-path problem to obtain a tight reformulation. Our computational results suggest that our contributions represent significant progress in solving instances of this problem.
\end{abstract}

\section{Introduction}

This paper addresses the dynamic lot-sizing problem with safety stocks and limited inventories which is to find the production/order policy so as to minimize the fixed setup, variable production and inventory holding costs over a given number of planning horizon. Instances of the problem often arise in practice, such as inventory, production, supply chain management, and several other problem settings that make basic cost tradeoffs between inventory holding costs and setup costs, under some restrictions of the inventory lower and upper bound limits, but they are generally difficult to solve to optimality .

Due to their importance in inventory control and production planning, the capacitated version of the dynamic lot-sizing problems have been widely studied [7][10][16], e.g., Shaw et al. ${ }^{1}{ }^{15}$ ] presented a dynamic programming algorithm for the single-item capacitated problem with piecewise linear production costs. Li-Kubo [9] reformulated the uncapacitated and capacitated multi-echelon problems, show that they can be solved effectively. Van Hoesel et al.$^{[17]}$ showed that polynomial approximation method that produces solutions with a relative deviation from optimality that is bounded by a constant do exist by presenting fully polynomial approximation schemes. Miller et al.$^{\left[{ }^{13\}}\right.}$ developed strong valid inequalities and discussed how to implement inequalities successfully in a branch-and-cut algorithm. Vanderbeck et al . ${ }^{[18]}$ discussed the models of lot-sizing with startup times and their complexity, then proposed further models. In contrast, the safety stock or limited inventory model can be found in few references in the literature, with some exceptions such as Loparic et al. [111] who dealt with the nondecreasing lower bounds on stocks, Bitran et al. ${ }^{[4]}$ who considered two approximation formulations for the single-item capacitated problem, provided relative error bounds and algorithms for solving them, and Belvaux et al. ${ }^{[2]}$ addressed some practical lot sizing problems contain the safety stock, modeling them as mixed integer programs. Gutiérrez et $a l .{ }^{[5]}$ considered a very special (i.e., no any setup cost) problem with special bounded inventory, devised a dynamic programming algorithm based on a new characterization.

* Student Member : Graduate School, Tokyo University of Mercantile Marine (1-6 Etchujima 2, Koto, Tokyo 135-8533)

**Non-Member : Tokyo University of Mercantile Marine (1-6 Etchujima 2, Koto, Tokyo 135-8533) 
The contributions of this paper are twofold: Due to valid inequalities developed in this paper a forward procedure and a backward procedure is devised, respectively, to deal with safety stocks and limited inventories; new properties to obtain the optimal policy are developed, to the best of our knowledge these properties are the first be proved in this paper. Based on these properties a dynamic programming algorithm is introduced, we then add prior covers to the branch-and -bound tree of the reduced shortest-path problem to obtain stronger formulation due to the provision of tight lower bounds. Using the benchmark problems of differing sizes available in literature the performance of this approach is tested.

The rest of this paper are outlined as follows: Firstly, Section 2 introduces the notation and the general mixed integer programming formulation. In Section 3 we propose an equivalent formulation due to two procedures dealing with safety stocks and limited inventories, and In Section 4 new properties that identify optimal policies are proved, then a shortest-path formulation is developed, and Section 5 discusses computational results. Finally, concluding remarks are offered in Section 6.

\section{Problem Description and Formulation}

The dynamic lot-sizing problem with safety stocks and limited inventories is stated as follows.

- Demands of each item are time independent and may occur at any period.

- Once an item is produced, a fixed setup cost occurs in addition to nonincreasing variable production cost.

- Inventory holding costs are computed based on the end-of-period inventory.

- There is an upper and lower bound on inventory for each item in each period.

- No backlogging of unsatisfied demand is permitted so that requirements must be met as they occur.

- Items reach immediately in any period whenever an order is placed, in other words lead times are negligible.
With the above assumptions that will hold throughout this paper, we determine a production schedule that minimizes the fixed setup, variable production and inventory holding costs within a rolling horizon framework.

To formulate the problem mathematically, we define the sets, parameters and decision variables as follows:

Sets

$P$ : the set of products/items.

\section{Parameters}

$T$ : the planning horizon, indexed as $1, \cdots, T$.

$f_{t}^{p}$ : the fixed setup cost which must be paid whenever an order of item $p$ is placed in period $t$.

$c_{t}^{p}$ : the variable production cost of item $p$ in period $t$, i.e., the unit cost of item $p$. According to assumptions defined above, for every $t=$ $1, \cdots, T-1$, we have $c_{t}^{p} \geq \mathrm{c}_{t+1}^{p}$.

$h_{t}^{p}$ : the positive unit cost of holding stock or inventory of item $p$ at the end of period $t$.

$d_{t}^{p}$ : the prespecified demand for item $p$ in period $t$.

ILB $B_{t}^{h}$ : the safety stock of item $p$ in period $t$.

$\overline{I U B_{t}^{p}}$ : the limited inventory of item $p$ in period $t$. $M_{t}$ : a large constant which must not limit feasible lot sizes of any period. It is enough that define $M_{t}=\sum_{p \in \mathrm{P}} \Sigma_{t=1}^{T} d_{t}^{p}$.

\section{Decision Variables}

$\psi_{t}^{p}$ : the continuous variable represents inventory or stock of item $p$ held over at the end of period $t$.

$x_{t}^{p}$ : the continuous nonnegative variable which represents the amount of item $p$ produced in period $t$.

$\delta_{t}^{p}$ : the binary variable indicates whether or not we produce item $p$ in period $t$

Thus, the mathematical programming formulation of the problem is as follows:

DLSSL:

$$
\begin{array}{ll}
\text { Min } & \sum_{p \in P} \sum_{t=1}^{T}\left(f_{t}^{p} \delta_{t}^{p}+c_{t}^{p} x_{t}^{p}+h_{t}^{p} \psi_{t}^{p}\right) \\
\text { s.t. } & \psi_{t-1}^{p}+x_{t}^{p}-\psi_{t}^{p}=d_{t}^{p}, \\
& \quad \forall p \in P, t=1, \cdots, T, \\
& x_{t}^{p} \leq M_{t} \delta_{t}^{p}, \quad \forall p \in P, t=1, \cdots, T, \\
& \underline{L I B_{t}^{p}} \leq \psi_{t}^{p} \leq \overline{I U B_{t}^{p},}
\end{array}
$$




$$
\begin{array}{ll} 
& \forall p \in P, t=1, \cdots, T, \\
x_{t}^{p}, \psi_{t}^{p} \geq 0, & \forall p \in P, t=1, \cdots, T, \\
\delta_{t}^{p} \in\{0,1\}, & \forall p \in P, t=1, \cdots, T .
\end{array}
$$

The objective minimizes the sum of the fixed setup, variable production and inventory holding costs for all items over the planning horizon of $T$ periods. Equation (1) is a set of inventory balance constraints that equate the supply of an item in a period with its demand or usage. In any period, the supply for an item is the inventory from the prior period $\psi_{t-1}^{p}$ (that is, preceding inventory), plus the production in the period $x_{t}^{p}$. This supply can be used to meet demand in period $d_{t}^{p}$, or held in inventory as $\psi_{t}^{p}$ (that is, succeeding inventory), and the constraints (2) guarantee that an appropriate setup cost is incurred whenever the production is positive. Constraints in (3) enforce limitations of the safety stock and limited inventory of any period.

\section{An Equivalent Formulation}

Before introduce an extended formulation, it is needed to define some appropriate transformations as follows:

At first, we offer the following valid inequalities to be theoretical grounds for the forward procedure to deal with the safety stock.

PROPOSITION 1 If in the problem (DLSSL) for the minimal feasible inventory of period $t$ and item $p,\left(\psi_{t}^{p}\right)_{\min },\left(\psi_{i}^{p}\right)_{\min } \geq \underline{I L B_{t-1}^{p}}-d_{t}^{p}$ is a family of valid inequalities.

ProOF. Since $x_{t}^{p} \geq 0$, Equation (1) implies

$$
\psi_{t}^{p} \geq \psi_{t-1}^{p}-d_{t}^{p}, \forall p \in P, t=1, \cdots, T .
$$

For $\psi_{t}^{p} \geq \underline{I L B_{t}^{p}}$, we have,

$$
\psi_{t}^{p} \geq \underline{I L B_{t-1}^{p}}-d_{t}^{p}, \forall p \in P, t=1, \cdots, T .
$$

This makes the following inequality hold. $\left(\psi_{t}^{p}\right)_{\min } \geq \underline{I L B_{t-1}^{p}}-d_{t}^{p}, \forall p \in P, t=1, \cdots, T$.

This observation is the basis for the forward procedure dealing with the non-negative safety stock of item $p$ in period $t$ given below:

FPSS (forward):

Step 1: Define the initial safety stock of an item as its initial inventory.

$$
\underline{I L B}_{t}^{p}=\psi_{t}^{p}, \quad \forall p \in P, t=0 .
$$

Step 2: The concept deals with the positive lower bounds on stocks similar to[2] is extended to treat the nonnegative safety stocks.

$$
\begin{array}{r}
\underline{I L B_{t}^{p}} \leftarrow \max \left\{\underline{I L B_{t}^{p}}, \underline{\left.I L B_{t-1}^{p}-d_{t}^{p}\right\},}\right. \\
\forall p \in P, t=1, \cdots, T .
\end{array}
$$

Step 3: Update the terminal safety stock by selecting the bigger one between the safety stock and required terminal inventory of each item.

$$
\underline{I L B_{t}^{p}} \leftarrow \max \left\{\underline{I L B_{t}^{p}}, \psi_{t}^{p}\right\}, \quad \forall p \in P, t=T .
$$

Step 4: Accordingly, the net demand $D_{t}^{p}$ and net inventory $\Psi_{t}^{p}$ for item $p$ in period $t$ are defined as:

$$
\begin{aligned}
D_{t}^{p} & \stackrel{\text { def }}{=}\left\lceil d_{t}^{p}+\underline{I L B_{t}^{p}}-\underline{I L B_{t-1}^{p}}\right\rfloor^{+}, \\
\psi_{t}^{p} & \stackrel{\text { def }}{=}\left\lceil\psi_{t}^{p}-\underline{I L B_{t}^{p}}\right\rfloor^{+} .
\end{aligned}
$$

Where, the initial net inventory $\Psi_{0}^{p} \equiv \psi_{0}^{p}-$ $\underline{I L B_{0}^{p}}=0$.

Then the limited inventory is considered. Theoretically, the following valid inequalities are needed.

PROPOSITION 2 If in the problem (DLSSL) for the maximal feasible net inventory of period $t$ and item $p, \quad\left(\Psi_{t}^{p}\right)_{\max }, \quad\left(\Psi_{t}^{p}\right)_{\max } \leq \overline{N I U B}_{t+1}^{p}+D_{t+1}^{p}$ is a family of valid inequalities.

PROOF. From Equations (1), we have

$$
\begin{aligned}
\Psi_{t}^{p}+x_{t+1}^{p} & =\Psi_{t+1}^{p}+D_{t+1}^{p} . \\
\forall p & \in P, t=1, \cdots, T-1 .
\end{aligned}
$$

Since $x_{t+1}^{p} \geq 0$,

$$
\begin{gathered}
\Psi_{t}^{p} \leq \Psi_{t+1}^{p}+D_{t+1}^{p} \leq{\overline{N I U B_{t+1}^{p}}}^{p}+D_{t+1}^{p} . \\
\forall p \in P, t=1, \cdots, T-1 .
\end{gathered}
$$

Clearly this implies valid inequalities

$$
\begin{aligned}
\left(\Psi_{t}^{p}\right)_{\max } & \leq \overline{N I U B}_{t+1}^{p}+D_{i+1}^{p}, \\
& \forall p \in P, t=1, \cdots, T-1 .
\end{aligned}
$$

This inequality holds.

Based on this inequality, a backward procedure that gives ways of treating the net limited inventory is outlined below:

BPLI (backward):

Step 1: Initialize the terminal net limited inventories of every item as follows: 


$$
{\overline{N I U B_{t}^{p}}}_{t}={\overline{I U B_{t}^{p}}}_{-\underline{I L B^{p}}}, \forall p \in P, t=T .
$$

Step 2: Update the non-negative net limited inventories of every item, for $t=1, \cdots, \mathrm{T}-$ 1 which indexs time periods, as follows:

$$
\begin{gathered}
\overline{\operatorname{NIUB}}_{t}^{p} \leftarrow \min \left\{\overline{\operatorname{NIUB}}_{t}^{p},{\overline{N I U B^{t}}}_{t+1}+D_{t+1}^{p}\right\}, \\
\forall p \in P, t=1, \cdots, T-1 .
\end{gathered}
$$

Step 3: Select the smaller one between the initial and first net limited inventories of each item.

$$
\begin{aligned}
& \overline{\text { IIUB }}_{t}^{p} \leftarrow \min \left\{\overline{N I U B}_{t}^{p}, \overline{\text { NIUB }}_{t+1}^{p}+D_{t+1}^{p}\right\}, \\
& \forall \Psi_{0}^{p} \leq \overline{N I U B}_{1}^{p}+D_{1}^{p}, p \in P, t=0 .
\end{aligned}
$$

PROPOSITION 3 If in the problem (DLSSL) deal with the safety stock and limited inventory, respectively by the forward procedure FPSS and backward procedure BPLI for some period t and any item $p$, the optimal strategy will not change ( $i$. $e .$, the lot sizes in the optimal solution is the same for both cost structures).

PROOF. Let $\mathrm{IR}_{+}^{3 T}$ denote the set of feasible solutions to DLSSL. Then the objective

$$
\begin{aligned}
& \min _{(x, \psi, o) \in \mathbb{R _ { t } ^ { 3 }}}\left\{\sum_{p \in P} \sum_{t=1}^{T}\left(f_{t}^{p} \delta_{t}^{p}+c_{t}^{p} x_{t}^{p}+h_{l}^{p} \psi_{t}^{p}\right)\right\} \\
& =\min _{(x, \psi+I L B, \delta) \in \mathbb{R}_{t}^{3 r}}\left\{\sum_{p \in P} \sum_{t=1}^{T}\left(f_{t}^{p} \delta_{t}^{p}+c_{t}^{p} x_{t}^{p}\right)\right. \\
& \left.+\sum_{p \in P} \sum_{t=1}^{T} h_{t}^{p}\left(\Psi_{t}^{p}+\underline{I L B_{t}^{p}}\right)\right\} \\
& =\min _{(x, \Psi+I L B, \delta) \in I R_{+}^{3 r}}\left\{\sum_{p \in P} \sum_{t=1}^{T}\left(f_{l}^{p} \delta_{t}^{p}+c_{l}^{p} x_{t}^{p}+h_{l}^{p} \Psi_{l}^{p}\right)\right\} \\
& +\sum_{p \in P} \sum_{t=1}^{T} h_{t}^{p}{\underline{I L B_{t}^{p}}}^{p}
\end{aligned}
$$

Then consider flow conservation constraints of the formulation DLSSL:

$$
\begin{aligned}
& x_{t}^{p}=\psi_{t}^{p}-\psi_{t-1}^{p}+d_{t}^{p} \\
& =\psi_{t}^{p}-\underline{I L B_{t}^{p}}+\underline{I L B_{t}^{p}}-\psi_{t-1}^{p}+\underline{I L B}_{t-1}^{p} \\
& -\underline{I L B_{t-1}^{p}+d_{t}^{p}} \\
& =\left\lceil\psi_{t}^{p}-\underline{I L B_{t}^{p}}\right\rfloor^{+}-\left\lceil\psi_{t-1}^{p}-\underline{I L B_{t-1}^{p}}\right\rfloor^{+}
\end{aligned}
$$

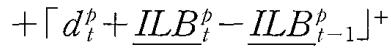

$$
\begin{aligned}
& =\psi_{t}^{p}-\psi_{t-1}^{p}+D_{t}^{p} .
\end{aligned}
$$

The set of constraints (3) are replaced by

$$
0 \leq \Psi_{t}^{p} \leq \overline{N I U B_{t}^{p}} \text {. }
$$

Therefore, when the safety stock and limited inventory is dealt with, respectively by the forward procedure FPSS and backward procedure BPLI the optimal lot sizes will not change.

Therefore, by the help of the forward procedure FPSS and backward procedure BPLI, the problem formulation DLSSL can be extended as the following equivalent formulation:

DLSSL'

$$
\begin{aligned}
\operatorname{Min} \sum_{p \in P} \sum_{t=1}^{T}\left(f_{t}^{p} \delta_{t}^{p}+c_{t}^{p p} x_{t}^{p}+h_{t}^{p} \Psi_{t}^{p}+h_{t}^{p} \underline{I L B_{t}^{p}}\right) & \\
\text { s.t. } \Psi_{t-1}^{p}+x_{t}^{p}-\Psi_{t}^{p}=D_{t}^{p} & \forall p \in P, t=1, \cdots, T, \\
x_{t}^{p} \leq M_{t} \delta_{t}^{p} & \forall p \in P, t=1, \cdots, T, \\
\Psi_{t}^{p} \leq \overline{N I U B_{t}^{p}} & \forall p \in P, t=1, \cdots, T, \\
\Psi_{0}^{p}=0 & \forall p \in P \\
x_{t}^{p}, \Psi_{t}^{p} \geq 0 & \forall p \in P, t=1, \cdots, T, \\
\delta_{t}^{p} \in\{0,1\} & \forall p \in P, t=1, \cdots, T,
\end{aligned}
$$

The objective function seeks to minimize the sum of the fixed setup, variable production and inventory holding costs over the planning horizon of $\mathrm{T}$ periods. Note the inventory holding costs consist of net inventory holding costs and a constant of total safety stocks costs. Equation (5) is a set of inventory balance constraints that equates the production of an item in a period with its demand or usage. Constraints in (6) guarantee that an appropriate setup cost is paid whenever a production occurs. Constraints in (7) enforce limitations of the limited inventory of each period. Finally, Equation (8) denotes the initial net inventory of an item is zero.

\section{Network Representation and Problem Reformulation}

\subsection{New Properties}

The question that remains to be answered is how to show the key observations to obtain a dynamic programming formulation of the original problem, or equivalently, how to show it suffices even when inventories are bounded to consider only feasible solutions in which the inventory at the ending of the prior periods of production periods is zero, a new production occurs only when the inventory goes to zero, this is called zero inventory ordering property. Here, the amount of products that is produced is exactly enough to meet the demand until the next time ( $i$. $e$, which is the next time when inventory goes to zero) that production is planned to occur.

PROPOSITION 4 In the problem (DLSSL') even when the inventory levels are bounded variables, 
for every item $p$ and in any period $t$, there exists an optimal policy such that production takes place only if inventory at the begin of the period is zero, i.e., $\Psi_{t-1}^{p} x_{t}^{p}=0$

PROOF. Suppose an order for item $p$ is placed in period $t^{*}$ before the previous order for $p$ which is placed in period $i\left(<t^{*}\right)$ is completely depleted, i.e., $\Psi_{t^{*}-1}^{p}>0$ and $x_{t^{*}}^{p}>0$, or $\Psi_{t^{*}-1}^{p} x_{t^{*}}^{p}>0$.

If such $t^{*}$ exists, a cheaper solution can always be obtained by increasing the lot size of period $t^{*}$ by $\Psi_{t^{*}-1}^{p}$ and decreasing the last order before period $t^{*}$ by the same amount, i.e.,

$$
\begin{aligned}
x_{t^{*}}^{p} & \leftarrow x_{t^{*}}^{p}+\Psi_{t^{*}-1}^{p}, \\
x_{i}^{p} & \leftarrow x_{i}^{p}-\Psi_{t^{*}-1}^{p},
\end{aligned}
$$

For this alteration does not incur any additional setup cost, does reduce variable production cost $\left\lceil\mathrm{c}_{i}^{p}-\mathrm{c}_{t^{*}}^{p}\right\rfloor^{+} \Psi_{t^{*}-1}^{p}(\geq 0)$ and does save the inventory $\left.\operatorname{cost} \Gamma\left(\sum_{s=i}^{t^{*}-1} h_{s}^{p}\right) \Psi_{t^{*}-1}^{p}\right\rfloor(>0)$, by $\Psi_{s}^{p} \leftarrow \Psi_{s}^{p}-\Psi_{t^{*}-1}^{p}, \forall s$ $=i, \cdots, t^{*}-1$. Moreover, this alteration does not increase any inventory level except decreasing some inventory levels during the interval $[i$, $\left.t^{*}-1\right]$.

Due to the decrement of inventory levels if there exists a period $t^{\prime *}$ whenever an order for item $p$ is placed in period $t^{*}$ before the previous order which is placed in period $i^{\prime}\left(<t^{\prime \prime} *\right)$ is completely depleted, i.e., $\Psi_{t^{*-1}-1}^{p} x_{t^{*}}^{p}>0$, then there always exists a more cheaper policy by a similar alteration that leads to $\Psi_{t^{* *}-1}^{p} x_{t^{* *}}^{p}=0$; otherwise, terminate. This alteration is iterated until no such a period $t^{*},\left(t^{* *}, t^{\prime \prime *}, \cdots\right)$ which satisfies $\Psi_{t^{*}-1}^{p} x_{t^{*}}^{p}>0$ exists.

Hence, $\Psi_{t-1}^{p} x_{t}^{p}=0, \forall \Psi_{t}^{p} \leq \overline{N I U B}_{t}^{p}, p \in P, t=$ $1, \cdots, T . i . e .$, even when the inventory levels are bounded, if an order is placed for item $p$ in time period $t$, then the conventional inventory for point $p$ at the end of period $t-1$ is zero. This property holds for all extreme points.

PROPOSITION 5 In the problem (DLSSL') for every $t$ and $p$ there exists an optimal policy such

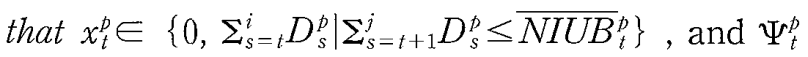
$\in\left\{0, \Sigma_{s=t+1}^{j} D_{t}^{p} \mid \Sigma_{s=t+1}^{j} D_{s}^{p} \leq \overline{N_{U} U B_{t}^{p}}\right\}$.

PROOF. Since all demands must be met, any value for $\Psi_{t^{*}}^{p} \not \subset\left\{0, \Sigma_{s=t^{*}+1}^{j} D_{s}^{p}\right\}$ or $x_{t^{*}}^{p} \notin\left\{\left\{0, \Sigma_{s=t^{*}}^{j} D_{s}^{p}\right.\right.$ \} implies there exists a period $t^{*}$ such that $\Psi_{l^{*}-1}^{p}$ $x_{t^{*}}^{p}>0$. It is clear that if this is true then two flows must cross somewhere which is a violation of the property of an extreme flows, that is to say among the optimal policy for DLSSL' no such a period $t^{*}$ to satisfy these conditions.

Moreover, Proposition 4 satisfies inventory upper bound limits $\Psi_{t}^{p} \leq{\overline{N I U B_{t}^{p}}}_{\text {, }}$ i.e. , the set of constraints (7) is not violated, this leads to

$$
\begin{aligned}
& \Psi_{t}^{p} \in\left\{0, \sum_{s=t+1}^{j} D_{s}^{p} \mid \sum_{s=t+1}^{j} D_{s}^{p} \leq \overline{N I U B_{t}^{p}},\right. \\
&\forall p \in P, 1 \leq t<j \leq T\}, \\
& x_{t}^{p} \in\left\{0, \sum_{s=t}^{j} D_{s}^{p} \mid \sum_{s=t+1}^{j} D_{s}^{p} \leq \overline{N I U B_{t}^{p},}\right. \\
&\forall p \in P, 1 \leq t \leq j \leq T\} .
\end{aligned}
$$

Since the linear relaxation of a shortest-path problem always has integer vertices, the significance of these propositions is that any solution with these properties corresponds to a path through the shortest-path network. Below we show how properties developed above can be exploited to obtain a tight reformulation, i.e., a reformulation with small linear programming relaxation gaps. A tighter problem reformulation can provide better lower bounds and help expedite convergence in exact branch-and-bound algorithms.

\subsection{Defining and Adding a Priori}

We now discuss issues that are important in the implementation of the network representation. This include building covers to be added a priori to the branch-and-bound tree.

To do this, we define $\sigma_{t}(p)$ to be the maximum period with the restriction of the net limited inventory for item $p$ in period $t$ such that a set of prior covers with respect to $\sigma_{t}(p)$ for the network representation is

$$
\left[t-1, \sigma_{t}(p)\right] .
$$

Where, a reasonable choice of $\sigma_{t}(p)$ is $\sigma_{t}(p) \stackrel{\text { def }}{=} \arg \max _{j=1, \cdots, T}\left\{\sum_{s=t+1}^{j} D_{s}^{p} \leq \overline{N I U B_{t}^{p}} \mid p \in P\right\}$

This has the effect of forcing the demand for item $p$ in interval $\left[t+1, \sigma_{\mathrm{t}}(p)\right]$ to be satisfied in period $t$.

Covers can be generated and added a priori not only at the root node but also at any other node of 
the branch-and-bound tree where some subsets of the prior covers yield the lower bound for that node. It is expected as well that they will be effective in reducing the number of variables and memory of requirement.

\subsection{Dynamic Programming}

Apply the dynamic programming algorithm to the dynamic lot-sizing problem with safety stocks and limited inventories. Let $F(j, p)$ denote the optimal cost to the problem to satisfy demands of $p$ through period $1,2, \cdots, j$. Assuming a regeneration point 0 to represent the time period immediately prior to the beginning of the planning horizon in which the cost is zero, i.e.,

$$
\mathrm{F}(0, p)=0 \text {, }
$$

the minimum cost of $p$ through period $j$ is equal to those through period $i(<j)$, plus the costs of ordering enough in period $i+1$ to satisfy demands from periods $i+1$ through $j, i . e ., f_{i+1}^{p}+c_{i+1}^{p}\left(\Sigma_{t=i+1}^{j}\right.$ $D_{t}^{p}+\sum_{s=i+1}^{j-1} h_{s}^{p}\left(\sum_{t-s+1}^{j} D_{t}^{p}\right)$.

Based on the properties devised above, the following forward recursion holds:

$$
\begin{aligned}
& F(j, p)=\min _{i \in\{0, \cdots, j-1:} \min _{\left.\left.j \in i+1, \cdots, \sigma_{i+1}(p)\right\}\right\}}\{F(i, p)+ \\
& \left.f_{i+1}^{p}+c_{i+1}^{p}\left(\sum_{t=i+1}^{j} D_{t}^{p}\right)+\sum_{s=i+1}^{j-1} h_{s}^{p}\left(\sum_{t=s+1}^{j} D_{t}^{p}\right)\right\} .
\end{aligned}
$$

The optimal cost of DLSSL' for $p$ can be obtained by calculating the forward dynamic programming recursion in sequence of $j=1,2, \cdots, T$.

\subsection{Problem Reformulation}

The dynamic programming algorithm can be reduced to a shortest-path problem. Create a regeneration point 0 and nodes $1,2, \cdots, T$ to denote time periods over the planning horizon, then let an arc $(i, j)$ of $p$ directly connect node $i$ through $j(>i)$. Define $C_{i j}$ as the cost of ordering enough in period $i+1$ to meet demand of $p$ for period $i+1$ through $j$, as the distance of arc $(i$, $j)$. Clearly, $\mathrm{C}_{i j}^{p}$ can be computed by

$$
\begin{aligned}
C_{i+1, j}^{p}=f_{i+1}^{p}+C_{i+1}^{p} & \left(\sum_{t=i+1}^{j} D_{t}^{p}\right) \\
& +\sum_{s=i+1}^{j-1} h_{s}^{p}\left(\sum_{t=s+1}^{j} D_{t}^{p}\right) .
\end{aligned}
$$

Finally, the shortest-path problem will be effective in solving the dynamic lot-sizing problem with safety stocks and limited inventories by the help of the above works we can reformulate
DLSSL, to yield an equivalent formulation: DLSSLR:

$$
\begin{aligned}
& \text { Min } \sum_{p \in P i j: j \in\left\{i+1, \cdots, \sigma_{i+1}(p)\right\}} C_{i+1, j}^{p} z_{i j}^{p} \\
& +\sum_{p \in P} \sum_{i} h_{i+1}^{p} \overline{I L B_{i+1}^{p}} \\
& \text { s.t. } \quad \sum_{j: j \in\left\{i+1, \cdots, \sigma_{i+1}(p)\right\}} z_{i j}^{p}-\sum_{j ; i \in\left\{j+1, \cdots, \sigma_{j+1}(p)\right\}} z_{j i}^{p} \\
& =\left\{\begin{array}{rl}
1 & i=\pi(p), p \in P, \\
0 & i \neq 0, \cdots, \pi(p), T, p \in P, \\
-1 & i=T, p \in P,
\end{array}\right. \\
& \pi(p) \equiv \arg \min _{t=0, \cdots, T-1}\left\{D_{t+1}^{p}>0 \mid p \in P\right\}, \\
& \quad z_{i j}^{p} \in\{0,1\}, \forall p \in P, i j=0, \cdots, T .
\end{aligned}
$$

Equation (10) models the flow balance for the underlying shortest-path problem for each item on nodes $0,1, \cdots, T$, where $z_{i, j}^{p}$ corresponds to the flow on the arc from node $i+1$ to node $j$. Equation (11) defines $\pi(p)$ to be the earliest period of an item which has a positive net demand. This deals with the condition that there may be some zero-demands of items appear at the first period.

Here we explore the relations between the general mixed-integer programming formulation DLSSL' (in $x, \Psi, \delta$ ) and the shortest-path formulation DLSSLR (in $z$ ) as follows:

$$
\begin{aligned}
\delta_{t}^{p} & =\sum_{j=t}^{\sigma_{t}(p)} z_{t-1, j}^{p}, \\
x_{t}^{p} & =\sum_{j=t}^{\sigma_{\ell}(p)}\left(\sum_{i=t}^{j} D_{i}^{p}\right) z_{t-1, j}^{p}, \\
\Psi_{t}^{p} & =\sum_{i=1}^{t}\left[\sum_{j=i}^{\sigma_{i}(p)}\left(\sum_{k=i}^{j} D_{k}^{p}\right) z_{i-1, j}^{p}-D_{i}^{p}\right] .
\end{aligned}
$$

The reformulation DLSSLR (in $z$ ) takes $O(\mid P$ $\left.\mid T^{2}\right)$ variables which is greater than $O(\mid P$ $\mid T$ ), that of the formulation DLSSL (in $x, \psi$, $\delta)$. Nevertheless, it is expected that our reformulation is much tighter than the original formulation, we will confirm this by computational experiments in the following section.

\section{Computational Results}

To test the efficiency computational experiments are performed on the general mixed-integer programming formulation and our reformulation.

The computations were carried out on a SONY', PCV-R70, Pentium II 500-MHz's PC with $128 \mathrm{Mb}$ of RAM, running under Windows 
Table 1 Computational results

\begin{tabular}{c|c|ccccccc}
\hline \multicolumn{2}{c|}{ Problem Name } & mtr6-15 & mtr12-15 & mtr24-15 & mtr6-30 & mtr12-30 & mtr24-30 & mt50-100 \\
\hline \multicolumn{2}{c}{ Number of Items } & 6 & 12 & 24 & 6 & 12 & 24 & 50 \\
\hline Number of Periods & 15 & 15 & 15 & 30 & 30 & 30 & 100 \\
\hline D & CPU Seconds & 4.78 & $10000.24+$ & $10000.87+$ & $10000.51+$ & $10000.95+$ & $10000.32+$ & $10000.50+$ \\
\cline { 2 - 9 } L & Memory & 388668 & 594016 & 1044476 & 602056 & 1048496 & 1929276 & 12088364 \\
\cline { 2 - 9 } S & Variables & 277 & 553 & 1105 & 547 & 1093 & 2185 & 15051 \\
\cline { 2 - 9 } S & Constraints & 367 & 733 & 1465 & 727 & 1453 & 2905 & 20051 \\
\cline { 2 - 9 } L & Best & 54488.6 & 116652 & 224535 & 129206 & 232364 & 427625 & 2745943 \\
\cline { 2 - 9 } & Bound & 54483.26 & 115792.31 & 216228.15 & 127019.43 & 213794.95 & 392770.37 & 2239829.34 \\
\hline D & CPU Seconds & 0.54 & 0.84 & 1.27 & 1.44 & 2.29 & 3.93 & 170.33 \\
L & Memory & 469068 & 738408 & 1304880 & 981248 & 1706856 & 2522936 & 17717300 \\
\cline { 2 - 8 } S & Variables & 241 & 473 & 959 & 705 & 1389 & 1993 & 14920 \\
S & Constraints & 97 & 193 & 385 & 187 & 373 & 745 & 5051 \\
L & Optimum & 54488.6 & 116443 & 221156 & 128904 & 226997 & 416677 & 2525058 \\
\cline { 2 - 8 } $\mathrm{R}$ & Bound & 54488.6 & 116443 & 221156 & 128904 & 226997 & 416677 & 2525058 \\
\hline
\end{tabular}

+: The loose formulation (DLSSL) was run for $10000 \mathrm{CPU}$ seconds without finding an optimal solution.

98. All the instances were solved with commercial programming software CPLEX, version 7.1. The test problems are generated from the benchmark lot-sizing problem LOTSIZELIB-trmd [12]. A time limit of 10000 seconds is used for each instance.

Table 1 shows that our expectation is confirmed by the computational experiments. Our results are summarized as follows: For larger problems the loose formulation DLSSL was run for $10000 \mathrm{CPU}$ seconds without finding an optimal solution, so far each of the gap

$$
\Delta Z=\left\lceil\frac{\text { Best }- \text { Lower Bound }}{\text { Lower Bound }}\right\rfloor \times 100(\%)
$$

was $0.74 \%, 3.84 \%, 1.72 \%, 8.69 \%, 8.87 \%, 22$. $60 \%$, respectively. The CPU time increase very rapidly with problem size. Nevertheless, our reformulation DLSSLR found optimal solutions within reasonable amounts of time for all of these test problems and all of the gaps are $0 \%$, moreover the growth rate with problem size is significantly slower, show that our reformulation DLSSLR is quite efficient. Numerical experiments indicate that the general mixed-integer programming formulation often requires exorbitant solution times for large-scale problems, but our reformulation is capable of solving quite large problem instances within a reasonable amount of time.

The shortest-path reformulation with added prior covers DLSSLR presented in this paper requires more variables, but it is perfectly tight, so it is quite efficient than the general mixed -integer programming formulation DLSSL. The results suggest that our contributions help to solve dynamic lot-sizing problems with safety stocks and limited inventories to optimality more efficiently than was before possible.

\section{Conclusions}

In this paper, the dynamid lot-sizing problem with safety stocks and limited inventories has been studied, a forward and backward procedure is devised, respectively to deal with safety stocks and limited inventories; new properties that identify optimal policies have been developed, these properties lead to an efficient dynamic programming algorithm that determines optimal policies of this problem. Then a priori is added to the branch-and-bound tree of the reduced shortest -path reformulation that produces extremely tight lower bounds. The framework proposed in this paper indicates that our contributions represent significant progress in solving instances of this problem.

\section{References}

[1] Bellman, R. E. Dynamic Programming, Princeton University Press. 1957.

[2] Belvaux, G. and L. A. Wolsey. Modeling practical lot-sizing problems as mixed integer programs. Management Science, 47 (7) : 993-1007. 2000.

[3] Blossom, A. P. The politics of lot sizing. 
Interfaces, 25(4): 60-65, 1995.

[4] Bitran, G. R. and H. Matsuo. Approximation formulation for the single-product capacitated lot size problem. Operations Research, 34(1): 63-73, 1986.

[5] Gutiērrez, J., A. Sedeño-Noda, M. Colebrook and J. Sicilia. A new characterization for the dynamic lot size problem with bounded inventory. Computers \& Operations Research, To appear.

[6] Karabuk, S., S. David Wu. Coordinating strategic capacity planning in the semiconductor industry. Technical Report, 99T -011, Lehigh University, Pennsylvania. 1999.

[7] Kubo Mikio. Logistics Engineering. ASAKURA Press, Tokyo. 2001 (in Japanese).

[8] Combinatorial Optimization and Algorithms. KYORITSU Press, Tokyo. 2000 (in Japanese).

[9] Li Shaorui and Mikio Kubo. Efficient formulations for the multi-echelon dynamic lot -sizing problem. 7th Academic Symposium between Japan and China Institute of Navigation, 75-82, Tokyo. 2001. , and An efficient formulation for multi-item dynamic lot-sizing problems. Journal of Japan Logistics Society, 10, To appear (in Japanese).

[11] Loparic, M., Y. Pochet, and L. A. Wolsey. The uncapacitated lot-sizing problem with sales and safety stocks. CORE Discussion Papers 9917 , Université Catholique de Louvain. 1999.

[12] Available on web at http://server. core. ucl. ac. be/wolsey/lotsizel.htm

[13] Miller, A. J., G. L. Nemhauser, and M. W. P. Savelesbergh. On the polyhedral structure for a multi-item production planning model with setup times. TLI White Papers, Georgia Institute of Technology. Atlanta. 2000.

[14] and Solving multi -item capacitated lot-sizing problems with setup times by branch-and-cut. TLI White Papers, Georgia Institute of Technology, Atlanta. 2000.
[15] Shaw, D. X. and A. P. M. Wagelmans. An algorithm for single-item capacitated economic lot sizing with piecewise linear production costs and general holding costs. Management Science, 44 : 831-838, 1998.

[16] Trigeiro, W. W., L. J. Thomas and J. O. McClain. Capacitated lot-sizing with set-up times, Management Science, 35:353-366, 1989.

[17] Van Hoesel, C. P. M. and A. P. M. Wagelmans. Fully polynomial approximation schemes for single-item capacitated economic lot-sizing problems. Mathematics of Operations Research. 26(2) : 339-357, 2001.

[18] Vanderbeck, F. Lot-sizing with start-up times. Research Papers in Management Studies, No. 22, Engineering Department, University of Cambridge, U. K. 1997.

[19] Wagner, H. M. and T. M. Whitin. Dynamic version of the economic lot size model. Management Science, 5:89-96, 1958 .

\section{質 疑 応 答}

岡山正人 (広島商船高専)：従来の研先のアプローチ と本研究のアプローチの違いはどこか、また従来 のアプローチの問題点のどこをこのアプローチで 解決できるのか。

李 少睿：従来の我々の提案していたモデル $[10]$ に 安全在庫と在庫上限制約を付加した点が本論文の 特徴です。本論文の結果を用いることによって実 際問題で不可欠な安全在庫や在庫上限制約を考慮 したロットサイズ決定が可能になります。

岡山正人：実際の現場では、こうした問題をどのよ うに解決しているのか。

李 少点: 現場の問題の解決についてMiller et al. [13], [14] はPhilips Electronics North America社 の容量制約付き問題を研究しています。Karabuk et al. ${ }^{[6]}$ は米国大手ウエハースメーカーに扔ける 生産能力計画を研究しています。Blossom ${ }^{[3]}$ は自 動車工場の問題を解決するための方策を議論して います。これらの事例のように、本論文のモデル は工場内の生産現場で使うことができます。 